\title{
ANALYSIS OF THE CAPABILITIES OF THE PROGRAMS FIJI, IPLAB AND DARFI IN THE STUDY OF DNA REPAIR ABILITIES IN THE CELLS OF PATIENTS WITH A MOSAIC FORM OF ATAXIA TELANGIECTASIA
}

\author{
Aleksandra Nozdracheva ${ }^{1^{*}}$, Roman Ushakov', Nadezhda Pleskach², Mirya Kuranova² \\ ${ }^{1}$ Peter the Great St. Petersburg Polytechnic University, Saint-Petersburg, Russia \\ ${ }^{2}$ Institute of Cytology of the Russian Academy of Science, Saint-Petersburg, Russia
}

\begin{abstract}
Different programs are used for processing and analyzing of fluorescent images. However, some of these programs produce uncertain results due to their dependence on human factor. Here we made a comparative study of three programs used for fluorescent images analysis: IPLab, Fiji and DARFI. The programs were compared by the fluorescence intensity measurement quality and cell nucleus area detection. We also analyzed the quality of counting of foci in the cell nucleus, the determination of foci area and foci fluorescence intensity. Fluorescent images were obtained with laser microscope Zeiss Axiovert 20OM after indirect immunofluorescence. The DNA repair study was performed on three cell lines from patients with ataxia-telangiectasia (AT). This disease is characterized by natural disruptions of the ATM kinase function. The study of the features of ATM kinase in DNA repair in patient cells is relevant not only in AT diagnostic, but also in the estimation of disease severity.
\end{abstract}

Keywords: IPLab, Fiji, DARFI, fluorescence microscopy, ataxia telangiectasia, DNA repair

\section{INTRODUCTION}

Currently, biological and biophysical studies use a variety of research methods, including immunofluorescence analysis, which is used, for example, to determine the qualitative and quantitative characteristics of intracellular antigens in cell cultures. Immunofluorescence analysis enables the visualization of the antigen after its binding with specific fluorescent antibodies. Some proteins, such as DNA repair proteins, microtubule and kinetochore organization centers, are localized in separate foci inside the cells. The study of the kinetics of the formation and elimination of foci is one of the most important and popular areas in the study of DNA repair. However, due to the small size of some protein formations and the ability of several foci to be located next to each other, data collection can be difficult. Therefore, it is important to have proper software for high-quality data acquisition from such images [1].

IPLab 3.6 is a software package for integrating microscope functions and image analysis developed by Scanalytics, which is currently part of BD Biosciences [2]. The most popular software for the processing of fluorescence images is an image processing package Fiji, ImageJ distribution that facilitates image analysis [3]. A relatively new program for the analysis of foci parameters is DARFI that uses the algorithm for automatic analysis of fluorescence images of cell nuclei with foci, including nucleus detection and foci detection [8].

Here we compared programs' capabilities to analyze the intensity of fluorescence of the cell nucleus, as well as the number, area and intensity of foci fluorescence inside the nucleus. Counting number of foci by the programs was compared to the manual counting performed by the user.

\section{OBTAINING CELL FOCI DATA USING IPLAB, FIJI, AND DARFI}

The analysis of fluorescence images often requires number of fluorescent foci counting. However, the questions are what to consider as focus and how large and bright a fluorescent spot should be in order to be taken into account. It is also important to define whether we deal with a chromatin structure or with nonspecific staining. For example, if we take into account the foci of smaller size, we may encounter the problem to differentiate them from the "noise" (nonspecific signal) in the nucleus.

The opposite case is the counting of foci clusters when several foci are located nearby [4]. Multiple and profound damages require special attention as they are often left unrepaired and result in cell death [5]. Double-strand breaks (DSBs) are important factor because they indicate serious problems in DNA structure. DSBs often happen in close mutual proximity and thus overlap with other types of DNA

\footnotetext{
*shurupchi@mail.ru
} 
damage. If it is not possible to analyze cluster foci using an optical partitioning system and a monochrome digital camera [9], it is better to analyze cluster foci manually. However, the problem of cluster foci criteria remains: should the cluster focus be considered as one large focus or as several foci? How many foci should be considered as cluster focus?

\subsection{Features of IPLab}

In this study, we revealed the subjective component in the IPLab program. When working with a fluorescent image of cells, it is important to properly select segmentation threshold depending on which certain (brighter) areas of the image are highlighted. While adjusting this parameter, researchers have to focus on their own vision of foci. Tuning of this parameter is difficult for cell nucleus in which, for example, many small foci are combined with cluster foci. In this case, the researcher has to decide whether to prefer better segmentation for cluster foci or for small individual foci. Due to the fact that big or cluster foci are much brighter than ordinary foci, small foci are not taken into account by the program. As a result, most small foci are lost.

IPLab has the ability to separate cluster and combined foci. However, it is also subjective because it fully depends on users' perception of the type of foci.

\subsection{Features of DARFI}

DARFI allows to count foci and their parameters specifically inside the nuclei. The program xbrindependently determines nuclei's boundaries, relying on an image of the nuclei colored with DAPI.
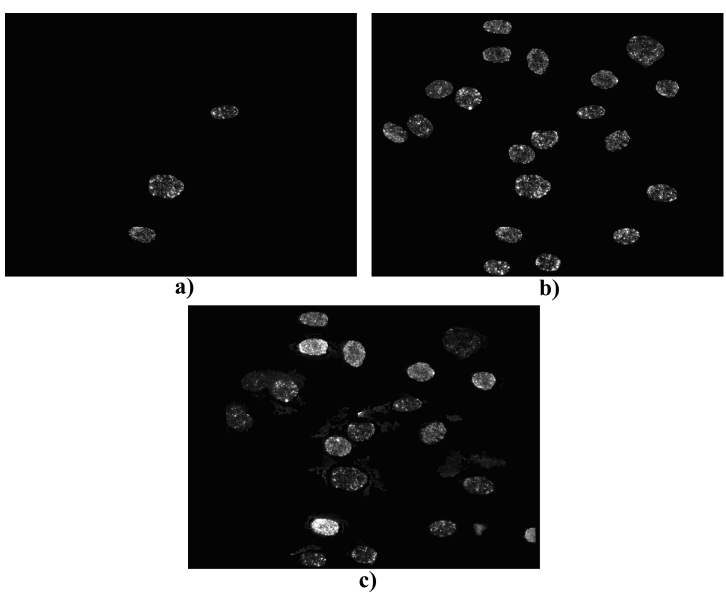

Figure 1. Highlighting of nuclei by DARFI on one image colored with DAPI, with the Sensitivity parameter equal to 6 (a), 9 (b) and 10 (c)

In the program settings, researchers can change: 1) sensitivity for nuclei stained with DAPI (see Figure 1); 2)nucleus size threshold, 3) foci sensitivity in the image with colored nuclei; 4) the percentage of filled foci area; 5) foci radius range, 6) the initial color of images (in DARFI, a researcher needs to convert the image into 8 bits), etc. Thus, it is possible to configure the program in order to process the foci of small size (which are difficult to distinguish with a naked eye), large size and clustered ones, but without "noise" being taken into account.

Depending on the specified settings, certain areas of the images will be highlighted: nuclei and foci with further calculation of the necessary parameters.

DARFI allows one to exclude cell nuclei that do not need to be analyzed (see Figure $2 \mathrm{a}, \mathrm{b}$ ), for example, if the program identified the background nonspecific signal as a nucleus. However, the program can highlight a nucleus and nonspecific background signal as one.
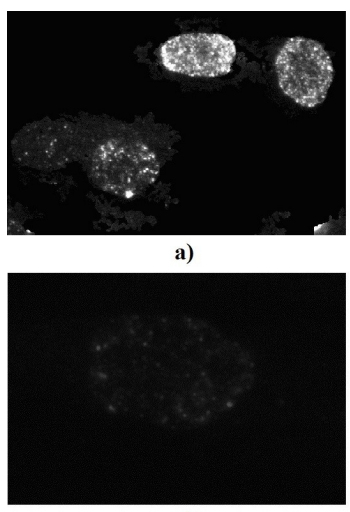

c)

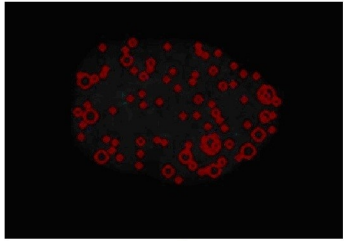

e)

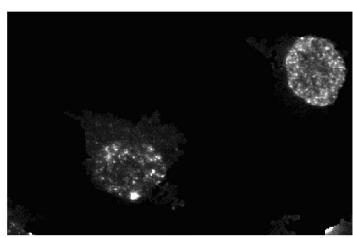

b)

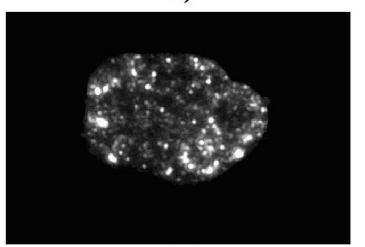

d)

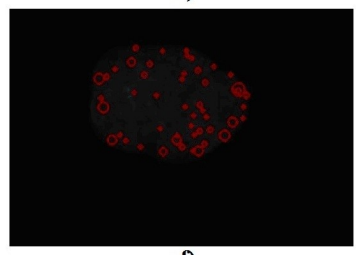

Figure 2. Highlighting of nuclei by DARFI on the same image section a) - before and b) - after exclusion of the highlighted nuclei of the cell. Image of one of the nuclei, c) - obtained with a Zeiss Axiovert 200M laser microscope; d) - Highlighting and processing of the selected nucleus by DARFI;

Highlighting of p53BP1 foci in this nucleus by DARFI with the value of the parameter Foci lookup sensitivity e) - 70 and

$$
\text { f) - 50, respectively }
$$

In addition to the number of DNA repair protein foci, important parameters of DNA damage are foci area and fluorescence intensity. DARFI allows to determine all of these. It also defines the area of cell nuclei and counts the fluorescence intensity of nuclei stained with DAPI or other dyes.

Evaluation of DARFI showed that it fails to recognize some nuclei (correctly manually detected). However, it recognizes foci of a very small size and with fluorescence intensity that does not differ much from the fluorescence intensity of the background of the nucleus. That is an advantage of the program as it is extremely difficult to recognize these foci with the naked eye. Therefore, the average number of foci per cell calculated using DARFI differs significantly from the average number of foci per cell calculated manually, or obtained with IPLab.

\subsection{Features of Fïi}

One of the algorithms in Fiji can highlight the nuclei using the formation of "masks" [6]. However, at this stage, errors can arise as each user sees the 
boundaries of nuclei in their own way. In addition, the accuracy of the procedure also depends on the characteristics of a monitor and external factors, such as a glare from sunlight on the monitor, etc. Fiji creates a "binary mask" based on an existing image with nuclei, which is an image in which the area of the nuclei is black, and the background is white [6]. A similar method of binarization of the image is used in DARFI [8], where it can be done automatically without losing time on adjusting settings as in Fiji. However, it turns out that this process in DARFI is less controlled.

In Fiji, after the masks are formed, the adjacent nuclei can be separated by choosing Process-> Binary$>$ Watershed buttons from the menu. However, for nuclei that are not round, the method fails as the program can split a whole core into parts. In this case, user can manually edit the wrong separation. Obviously, these manipulations make the result more subjective. The stage of adjusting the contrast of the background is entirely done by the user that also adds subjectivity to the image processing. Indeed, the background of the image should be completely black, while the nuclei were the only relatively bright objects. The adjustment process on the Brightness/Contrast diagram is done manually.

Fiji program has the ability to count the number of foci in cells using the Find Maxima function. However, users must set the Noise tolerance parameter on their own, based on their vision of the foci, which also introduces subjectivity into the process of taking data from a fluorescent photo. In DARFI, you can change the sensitivity to find objects in a photo with a similar subjective component.

\subsection{Comparative characteristics of IPLab, Fïi and} DARFI for DNA damage analysis

When using the method of indirect immunofluorescence, it is important to find both the mean value of fluorescence intensity and its distribution within the cell. This approach allows to see the homogeneity of the cell population, as well as to identify peaks of population intensity [7].

We analyzed the fluorescence intensity of DAPI and HP1Y in the nuclei of dermal fibroblast line (N8SP) from the 23-year-old healthy donor using DARFI and the algorithm of Fiji mentioned above. For DARFI analysis, all images were converted into 8-bit format. When we changed the format in different versions of the graphic editor (GIMP 2.8 and GIMP 2.10), we noticed a significant difference in intensity between two images. The difference in the distribution of fluorescence intensity between two programs is shown in Figure 3. On the contrary, the analysis of $\mathrm{HP} 1 \mathrm{Y}$ fluorescence intensity distribution with Fiji software showed only one cell population (see Figure 3).

The biggest disadvantage of image analysis is its subjectivity. IPLab contains the highest subjective component in the calculation of the foci number, while DARFI and Fiji are less subjective and give the possibility to change the parameters to analyze different fluorescent images. However, DARFI requires an external tool to prepare an image (conversion to 8-bit color format) that can influence an analysis.

Depending on the task, a researcher can use a particular program or their combination. According to the results of our analysis, Fiji is most suitable for the measurement of fluorescence intensity of nuclei stained with DAPI or other fluorescent markers. Fiji is also suitable for counting the foci. However, DARFI's interface is simpler, which can help if the user does not know specific Fiji algorithms needed to count foci. Moreover, DARFI avoids subjectivity by giving an option to set up necessary parameters for foci analysis.
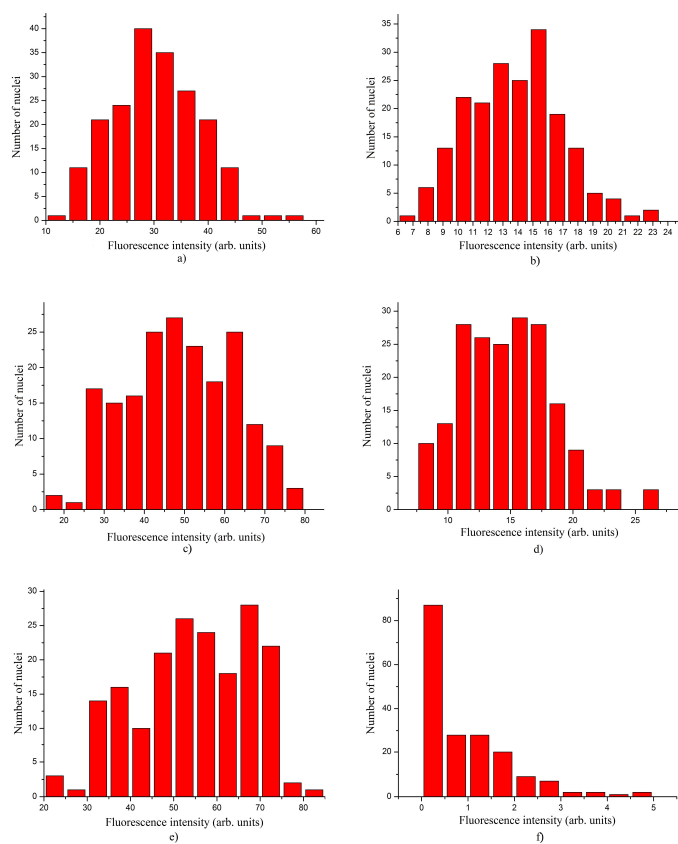

Figure 3. The distribution of the fluorescence intensity of DAPI (a, c, e) and HP1y (b, d, f) in the N8SP line, determined using: a, b) DARFI after conversion to 8-bit image in GIMP 2.8; c, d) DARFI after conversion to 8-bit image in GIMP 2.10; d, f) Fiji

\section{DNA REPAIR ABILITIES IN THE CELLS OF PATIENTS WITH A MOSAIC FORM OF ATAXIA TELANGIECTASIA}

Ataxia telangiectasia (AT) is a hereditary multisystem neurodegenerative disease caused by an autosomal recessive mutation in the ATM gene. This disease is characterized by chromosomal breakdown, a high risk of carcinogenesis and the sensitivity to ionizing radiation. AT is a polymorphic disease and can be hard to diagnose, especially in its mild or suppressed form. There is also an ataxiatelangiectasia-like disorder or ATLD which occurs due to mutations in the MRE11 gene. MRE11 encodes a double-strand break repair protein Mre11, which is a part of the MRN complex that also includes Rad5o and Nbs1 proteins.

\subsection{Cell lines}

The study of the dynamics of DNA repair was carried out on dermal fibroblast cell lines obtained from three patients with a clinical diagnosis of AT and confirmed mosaic expression of the active form of the ATM kinase pATM ${ }^{\text {Ser1981. }}$. All patients have an erased form of AT that requires detailed diagnosis.

NGS sequencing was performed for the AT9SP, AT10SP, and AT13SP cell lines to search for pathogenic substitutions in the ATM, MRE11A, RAD50, NBS1, CETX, APTX, and BRAT1 genes. In the case of the 
AT13SP line, a heterozygous compound mutation was detected in the ATM gene on Chr11: $108115727 \mathrm{C}>\mathrm{T}$ NM_o00051.3 (ATM): c.875C > T (p.Pro292Leu) chr11: 108196236_108196237delCT NM_oooo51.3 (ATM): c.6772_6253_6773_6773_2773 13). In all other cases, no obvious pathogenic substitutions were detected. However, detected substitutions and their combinations require further analysis for possible pathogenicity. Cell line from a 23-year-old healthy donor N8SP was used as a control.

\subsection{Methods}

To evaluate the activity of the active form of kinase

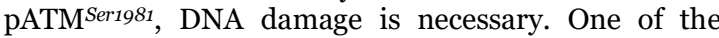
factors that damage DNA and create double-strand breaks is ionizing radiation. Cell cultures were exposed to X-ray at a dose of 2 Gy using an X-ray apparatus 150/300-14.

Foci detection were made with immunofluorescence analysis. All cell lines were obtained in the laboratory of cell metabolism and signaling at the Institute of Cytology of the Russian Academy of Sciences. Getting the primary dermal fibroblast lines of studied patients and control were carried out by the migration method using a Minimum Essential Medium (MEM, Gibco, USA) supplemented with $15 \%$ fetal bovine serum (Gibco, USA), 100 units $/ \mathrm{ml}$ penicillin, $100 \mu \mathrm{g} / \mathrm{ml}$ streptomycin (Gibco, USA), $7.5 \%$ sodium bicarbonate solution and 0.3 $\mathrm{mg} / \mathrm{ml} \mathrm{L}$-glutamine at $37{ }^{\circ} \mathrm{C}$ in an atmosphere with $5 \%$ $\mathrm{CO}_{2}$.Cells were grown on cover glasses in Petri dishes and then were washed with a phosphate-buffered saline (PBS), fixed with $3.7 \%$ formaldehyde solution diluted in PBS on ice at a temperature of $+4^{\circ} \mathrm{C}$, after which fixation was made with an ice $70 \%$ ethanol solution for 2 hours at room temperature, after washing with PBS, they were sterilized with $1 \%$ Triton solution (Triton-X-100) for 10 minutes at room temperature. Next, the cells were washed with PBS 3 times for 5 minutes and incubated in a blocking solution of $5 \%$ bovine serum albumin (BSA) diluted in PBS for 30 minutes at room temperature, after which they were washed with PBS. The first antibodies were mouse immunoglobulin (IgG) against the pATM ${ }^{\text {Ser198 }}$ (Merck Millipore, USA) and rabbit IgG against the p53BP1 (Abcam, UK). Goat IgG against mouse and rabbit $\operatorname{IgG}$ conjugated with AlexaFluor 488 and 546 (Invitrogen, USA) was used as second antibodies. Cells

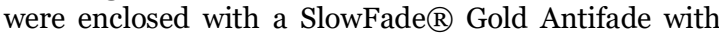
DAPI (Invitrogen, USA).

Fluorescent images ware obtained using a laser microscope ZeissAxiovert 200M.

\subsection{Results}

In all cell lines, the dynamics of the kinase reparation activity was studied by the estimation of pATM $^{\text {Ser1981 }}$ phosphorylation at different time points after exposure to ionizing radiation at a 2 Gy dose. The time points were $30 \mathrm{~min}, 1.5 \mathrm{~h}, 6$ and $24 \mathrm{~h}$.

The repair curves were constructed using the following parameters: the average number of foci per cell (see Figure 4 and 5), the average value of the fluorescence intensity of the foci per cell (see Figure 6 and 7) and the average value of the foci area per cell (see Figure 8 and 9). Also, the average number of foci
pATM ${ }^{\text {Ser1981 }}$ and p53BP1 per cell was counted manually (see Figure 10 and 11).

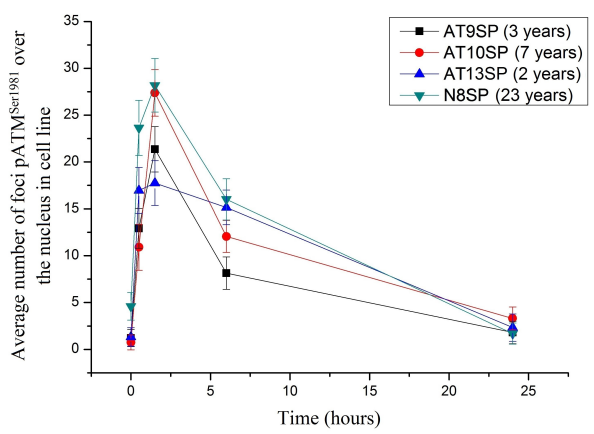

Figure 4. Dynamics of DNA repair by the average number of pATM $^{\text {Ser1981 }}$ foci per cell in the studied cell lines

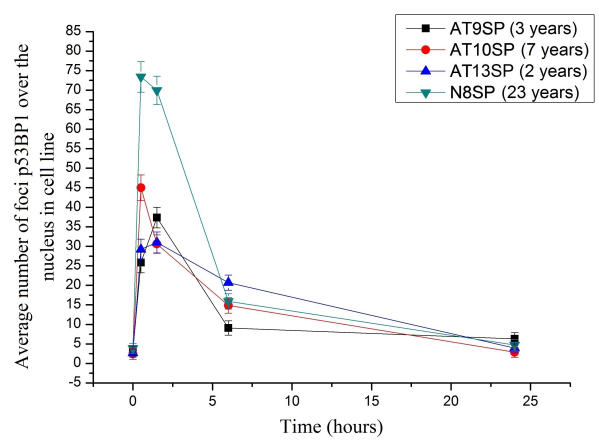

Figure 5. Dynamics of DNA repair by the average number of p53BP1 foci per cell in the studied cell lines

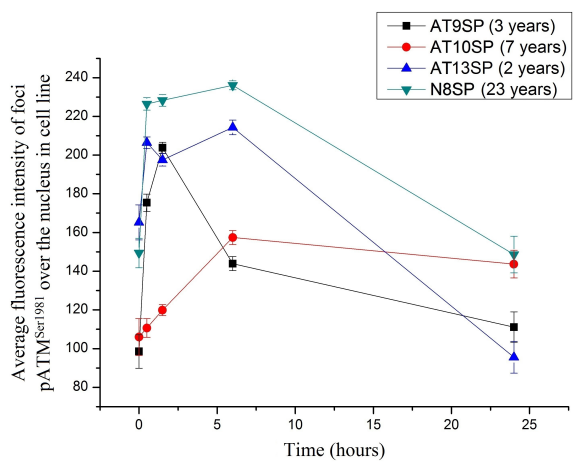

Figure 6. Dynamics of DNA repair by the average value of the fluorescence intensity of pATMSer1981 foci per cell in the studied cell lines 


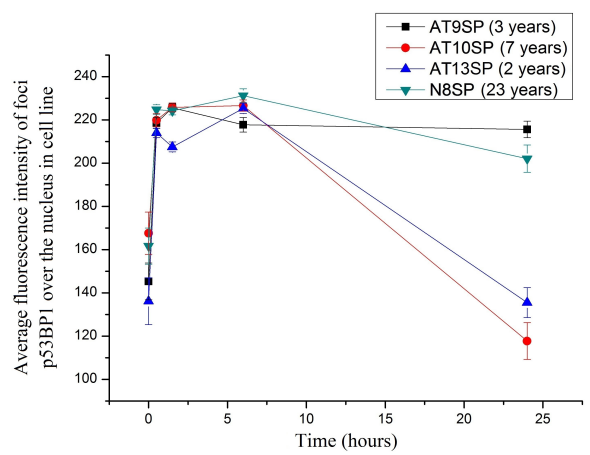

Figure 7. Dynamics of DNA repair by the average value of the fluorescence intensity of $\mathrm{p}_{53} \mathrm{BP} 1$ foci per cell in the studied cell lines

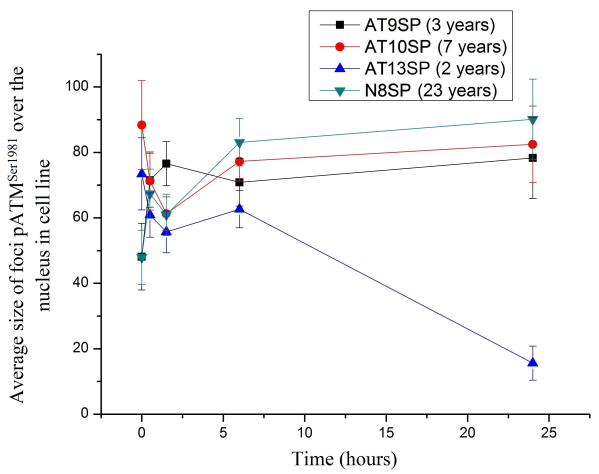

Figure 8. Dynamics of DNA repair by the average value of the foci area pATM ${ }^{\text {Ser1981 }}$ per cell in the studied cell lines

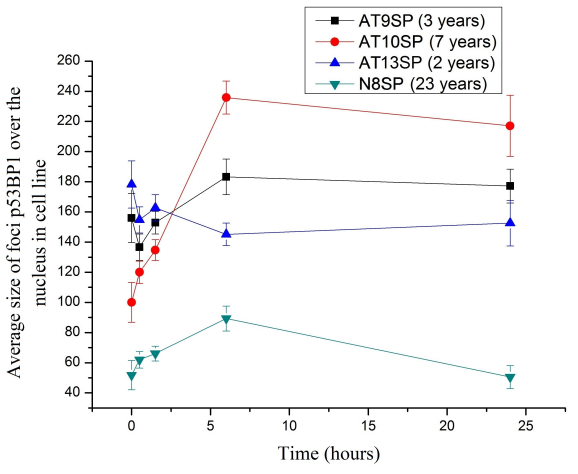

Figure 9. Dynamics of DNA repair by the average value of the foci area $\mathrm{p} 53 \mathrm{BP} 1$ per cell in the studied cell lines

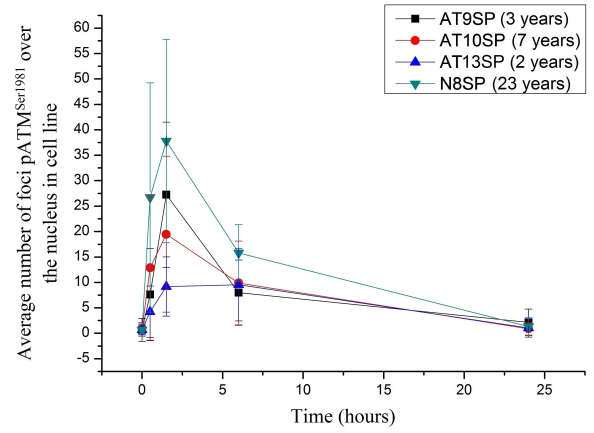

Figure 10. Dynamics of DNA repair by the average number of

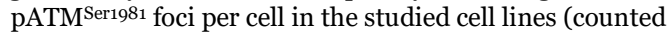

manually)

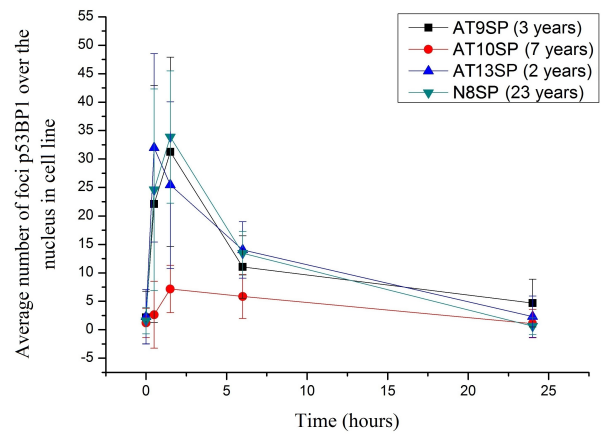

Figure 11. Dynamics of DNA repair by the average number of p53BP1 foci per cell in the studied cell lines (counted manually)

\section{CONCLUSION}

To study the features of DNA repair in the studied cell lines, the capabilities of the Fiji, IPLab and DARFI were tested. During the program testing for the correct analysis of foci parameters, the subjectivity of IPLab was discovered.

Fiji is best suited for measuring the fluorescence intensity of nuclei colored with DAPI or other fluorescent markers. Number of foci calculation's precision in Fiji depends on a user's acquaintance with certain algorithms, so DARFI is much easier to use. DARFI also allows users to get more accurate parameters of foci with a lower degree of subjectivity.

Based on the capabilities of the Fiji and DARFI programs, repair curves for the cells of patients with AT and a healthy donor were made and analyzed. The advantage of counting with programs over manual counting was demonstrated.

The data of DNA repair in the studied cell lines and comparison with the patients' cell picture showed significant differences in the fluorescence intensity, area and number of pATM ${ }^{\text {Ser1981 }}$ and p53BP1 foci between a healthy donor and patients with AT. Differences in foci parameters between AT cell lines depending on the severity of the form of the disease were also demonstrated. The results showed that the fluorescence intensity and foci area are also very important for the most complete understanding of DNA repair capabilities.

For example, it was shown that the foci area allows to judge about the severity of DNA damage: the larger foci are, the severe the damage. The cell line of the patient, who initially had a diagnosis of ATLD, which differs from the AT, showed the smallest foci area.

The number of foci and their appearance after DNA damage are also important indicators of normal DNA repair. The maximum number of foci pATM $^{\text {Ser1981 }}$ and p53BP1 was demonstrated by healthy donor dermal fibroblast cells at a time interval of 30 minutes after DNA damage.

Despite the fact that the patients had a mild or erased form of the disease, their cells had a significant delay in the response to DNA damage. 
Acknowledgements: The paper is a part of the research supported by the RFBR grant No. 18-315o0213. The authors would like to thank Aleksandr Morozov and Anastasia Demidova for the assistance in working with programs.

\section{REFERENCES}

1. E. Ledesma-Fernández, P. H. Thorpe, "Fluorescent foci quantitation for high-throughput analysis," J. Biol. Methods, vol. 2, no. 2, Jul. 2015.

DOI:10.14440/jbm.2015.62 PMid: 26290880 PMCid: PMC4538797

2. J. M. Shillingford, IPLab Imaging Software for Microscopy from BD Biosciences version 4.0, Biocompare, South San Francisco (CA), USA, 2010. Retrieved from:

https://www.biocompare.com/ProductReviews/41450-IPLab-Imaging-Software-ForMicroscopy-from-BD-Biosciences/; Retrieved on: Aug. 22, 2019

3. J. Schindelin et al., "Fiji: An open-source platform for biological-image analysis," Nat. Methods, vol. 9, no. 7, pp. 676 - 682, Jun. 2012

DOI: $10.1038 /$ nmeth.2019

PMid: 22743772

PMCid: $\mathrm{PMC}_{3855844}$

4. A. Sollazzo et al., "Live Dynamics of 53BP1 Foci Following Simultaneous Induction of Clustered and Dispersed DNA Damage in U2OS Cells," Int. J. Mol. Sci., vol. 19, no. 2, Feb. 2018.

DOI: $10.3390 /$ ijms19020519

PMid: 29419809 PMCid: PMC5855741

5. E. Bobkova et al., "Recruitment of 53BP1 Proteins for DNA Repair and Persistence of Repair Clusters Differ for Cell Types as Detected by Single Molecule Localization Microscopy," Int. J. Mol. Sci., vol. 19, no. 12, Nov. 2018

DOI: $10.3390 /$ ijms19123713

PMid: 30469529

PMCid: PMC6321197
6. T. Ferreira, W. Rasband, ImageJ User Guide - IJ 1.46r, National Institutes of Health, Bethesda (MD), USA, 2012.

Retrieved from:

https://imagej.nih.gov/ij/docs/guide/user-guide.pdf; Retrieved on: Aug. 22, 2019

7. M. L. Kuranova, N. M. Pleskach, T. A. Ledashcheva, V. M. Mikhel'son, I. M Spivak, "Mosaic forms of ataxia-telangiectasia," Tsitologiia, vol. 56, no. 8, pp. 619 - 629, 2014.

DOI: $10.1134 / \mathrm{S} 1990519 \mathrm{X} 15010058$

PMid: 25697008

8. И. В Озеров, "Математическое моделирование процессов индукции и репарации двунитевых разрывов ДНК в клетках млекопитающих при действии редкоионизирующего излучения с различной мощностью дозы,” к. ф.-м.н., МГУ имени М. В. Ломоносова, Радиобиология, Москва, Российской Федерации, 2015.

(I. V. Ozerov, "Mathematical modeling of the processes of induction and repair of double-stranded DNA breaks in mammalian cells after the action of rarely ionizing radiation with different dose rates," Ph.D dissertation, Lomonosov Moscow State University, Dept. of Radiobiology, Moscow, Russia, 2015.)

Retrieved from:

http://www.bio.msu.ru/res/Dissertation/675/DOC_FI LENAME/Ozerov avtoref.pdf;

Retrieved on: Aug. 22, 2019

9. М.Г. Заднепрянец и др., "Влияние физических характеристик ускоренных тяжёлых ионов на формирование и репарацию двунитевых разрывов ДНК," Писъма в ЭЧАЯ., том. 15, но. 6(218), стр. 563 - 572, 2018.

(M. G. Zadnipryanets, "The effect of the physical characteristics of accelerated heavy ions on the formation and repair of double-stranded DNA breaks," Letters in JINR, vol. 15, no. 6(218), pp. 563 - 572, 2018.)

Retrieved from:

http://www1.jinr.ru/Pepan_letters/panl_2018 6/17_ z adnepryan.pdf;

Retrieved on: Aug. 22, 2019 